\title{
ELECTROMECHANICAL ANALYSIS OF PIEZOELECTRIC LAMINATED COMPOSITE BEAMS
}

Ming-Hung Hsu

Department of Electrical Engineering, National Penghu University, hsu@npu.edu.tw

Follow this and additional works at: https://jmstt.ntou.edu.tw/journal

Dart of the Electrical and Computer Engineering Commons

\section{Recommended Citation}

Hsu, Ming-Hung (2005) "ELECTROMECHANICAL ANALYSIS OF PIEZOELECTRIC LAMINATED COMPOSITE BEAMS,"

Journal of Marine Science and Technology. Vol. 13: Iss. 2, Article 10.

DOI: $10.51400 / 2709-6998.2115$

Available at: https://jmstt.ntou.edu.tw/journal/vol13/iss2/10

This Research Article is brought to you for free and open access by Journal of Marine Science and Technology. It has been accepted for inclusion in Journal of Marine Science and Technology by an authorized editor of Journal of Marine Science and Technology. 


\section{Short Paper}

\section{ELECTROMECHANICAL ANALYSIS OF PIEZOELECTRIC LAMINATED COMPOSITE BEAMS}

\begin{abstract}
Key words: piezoelectric laminated composite beam, vibration characteristics, electromechanical coupled system, various boundary conditions.
\end{abstract}

Ming-Hung Hsu

\begin{abstract}
This work demonstrates the electromechanical behavior of piezoelectric laminated composite beams using the differential quadrature method (DQM). The Chebyshev-Gauss-Lobatto sample point equation is used to select the sample points. The electromechanical responses of piezoelectric laminated composite beams with various boundary conditions are determined. The transient responses of the derived systems are calculated using the Wilson-method. In this approach, only nine sample points are required to obtain convergence. Numerical results indicate that the DQM is valid for a piezoelectric laminated composite beam formulation.
\end{abstract}

\section{INTRODUCTION}

To extend the engineering applications for piezoelectric materials, the composite structure is preferred in future design concept. With the development of stable responses and high performance piezoelectric laminated composite beams have become very important. Piezoelectric material has been extensively applied in sensors and actuators. Wu [33] presented a general approach for examining the electromechanical responses of piezoelectric materials. Huang and Dai [15] proposed a theoretical model to examine the static and dynamic electromechanical responses of piezoelectric transducers that are treated as a beam. Chen et al. [10] studied the dynamic instability problems of a composite beam with piezoelectric material layers subjected to periodic axial compressive loads. Weinberg [32] solved a closed form solution for bending of Euler Bernoulli

Paper Submitted 10/07/04, Accepted 05/05/05. Author for Correspondence: Ming-Hung Hsu. E-mail: hsu@npu.edu.tw.

*Department of Electrical Engineering, National Penghu University piezoelectric beam and neglected the effect of transverse shear on the deformation of the beam. Tadmor and Kosa [28] extended the Weinberg analysis to materials with arbitrary electromechanical coupling coefficients by accounting for the variation of electric field in the beam layers. Yang and Huang [34] investigated a three-dimensional, infinitely extended, anisotropic piezoelectric solid containing a flat ellipsoidal crack with emphasis laced on when one of the principal axes of the crack becomes zero. Saitoh and Koshiba [23] formulated a stress method considering piezoelectric effects based on the finite element method, which can be applied to arbitrarily anisotropic material based optical wave-guide devices. In this work, the electromechanical behavior equations of the piezoelectric laminated composite beam are derived using the differential quadrature method (DQM). The calculated accuracy and integrity of this problem solved using DQM is demonstrated by a series of case studies.

\section{DIFFERENTIAL QUADRATURE METHOD}

There are many computational methods available for electromechanical analysis. In this work, the electromechanical responses of the piezoelectric laminated composite beams are investigated using the DQM. Bellman and Casti [1] and Bellman et al. [2] proposed originally DQM. This method reduces the partial differential equations into a set of algebraic equations and has been used extensively to solve a variety of problems in different fields of science and engineering. The DQM has been shown to be a powerful contender in solving initial and boundary value problems and has thus become an alternative to the existing methods. One of the fields among which one can find extensive DQM applications is structural mechanics. Bert and Malik [46] and Bert et al. [3, 7, 8] analyzed the static and free vibration of beams and rectangular plates using the 
DQM. Jang et al. [16] proposed the technique. The boundary points are chosen at a small distance. The technique can apply to the double boundary conditions of plate and beam problems. The cannot be enlarged for solution accuracy. The solutions oscillate when the is too small. Wang and Bert [31] took the boundary conditions into account in the DQ coefficients. Malik and Bert [19] solved the free vibration of the plates and showed that the boundary conditions can be built into the DQ coefficients. In the formulation, the multiple boundary conditions are directly applied to the DQ coefficients and thus it is not necessary to select a nearby point as the $\delta$-interval method does. In other words the accuracy of the calculated results will be independent of the value of $\delta$-interval. The DQ coefficients can be obtained by multiplication of the inverse matrix. Quan and Chang [21, 22] derived the weighting coefficients in a more explicit way. The explicit formulae are more convenient. Sherbourne and Pandey [24] solved buckling problems using the DQM. Feng and Bert [14] solved the flexural vibration analysis of a geometrically nonlinear beam using the DQM. Liew et al. [18] presented the analysis of the rectangular plates resting on Winkler foundations using the DQM. Liew et al. [17] presented the static analysis of laminated composite plates subjected to transverse loads using the DQM. Du et al. [13] used DQM for buckling analysis. Shu and Du [25] solved the free vibration of laminated composite cylindrical shells using the DQM. Tomasiello [30] solved initial-boundary-value problems using the DQM. Sun and Zhu [27] solved the incompressible viscous flow using the DQM. Choi et al. [12] solved the dynamic characteristics of a spinning Timoshenko beam using DQM. Tanaka and Chen [29] applied the DQM to the second-order time derivative in the elastodynamic formulations. The differential quadrature element method is used to discretize the differential equations and to analyze the structure problems with discontinuous loading and discontinuous boundary. Striz et al. [26] used the quadrature element method for static analysis. Chen [9] presented the application of the differential quadrature element method for the warping torsion bar model. Chen et al. [11] successfully solved two dimensional plane stress and plate bending problems using the quadrature element method. From what we mentioned above, it is undoubtedly that for the past twenty years, the DQM had been used extensively as an effective way to solve a variety of problems in different fields of science and engineering.

The efficiency and accuracy of the Rayleigh-Ritz method are dependent on the number and accuracy of the selected comparison functions. DQM does not involve such a difficulty, in terms of selecting the suitable comparison functions. The core of the DQM is that the derivative of a function at a sample point is approximated by a weighted linear sum of the functional values at all the points sampled in the domain. This method approximates the $m^{\text {th }}$ order partial derivate of $f(x, t)$ with respect to $x$ as the following equation:

$$
\left.\left.\frac{\partial^{m}}{\partial x^{m}}\left\{\begin{array}{c}
f\left(x_{1}, t\right) \\
f\left(x_{2}, t\right) \\
\vdots \\
\vdots \\
f\left(x_{N}, t\right)
\end{array}\right\} \cong\left[D_{i j}^{(m)}\right]\right\} \begin{array}{c}
f\left(x_{1}, t\right) \\
f\left(x_{2}, t\right) \\
\vdots \\
\vdots \\
f\left(x_{N}, t\right)
\end{array}\right\}
$$

for $i, j=1,2, \ldots, N$

where $f\left(x_{i}, t\right)$ is the functional value at the sample point $x_{i}$, and $D_{i j}^{(m)}$ are the DQ coefficients of the $m^{t h}$ order differentiation attached to these functional values.

The DQ coefficients are difficult to obtain, because they may be ill conditioned. Quan and Chang [21, 22] established a set of algebraic expressions to calculated the DQ coefficients to overcome the numerical illconditioning in determining the DQ coefficients $D_{i j}^{(m)}$, namely:

$$
f(x, t) \cong \sum_{i=1}^{N} \frac{M(x)}{\left(x-x_{i}\right) M_{1}\left(x_{i}\right)} f\left(x_{i}, t\right)
$$

where

$$
\begin{aligned}
& M(x)=\prod_{j=1}^{N}\left(x-x_{j}\right) \\
& M_{1}\left(x_{i}\right)=\prod_{j=1, j \neq i}^{N}\left(x_{i}-x_{j}\right) \text { for } i=1,2, \ldots, N
\end{aligned}
$$

Substituting equation (2) into equation (1) yields:

$$
D_{i j}^{(1)}=\frac{M_{1}\left(x_{i}\right)}{\left(x_{i}-x_{j}\right) M_{1}\left(x_{j}\right)} \text { for } i, j=1,2, \ldots, N \text { and } i \neq j
$$

and

$$
D_{i j}^{(1)}=-\sum_{j=1, j \neq i}^{N} D_{i j}^{(1)} \text { for } i=1,2, \ldots, N
$$

The second-order and higher-order derivatives of the DQ coefficients can also be obtained by matrix multiplication [19], thus:

$$
\begin{aligned}
& D_{i j}^{(2)}=\sum_{k=1}^{N} D_{i k}^{(1)} D_{k j}^{(1)} \text { for } i, j=1,2, \ldots, N \\
& D_{i j}^{(3)}=\sum_{k=1}^{N} D_{i k}^{(1)} D_{k j}^{(2)} \text { for } i, j=1,2, \ldots, N \\
& D_{i j}^{(4)}=\sum_{k=1}^{N} D_{i k}^{(1)} D_{k j}^{(3)} \text { for } i, j=1,2, \ldots, N
\end{aligned}
$$




$$
D_{i j}^{(4)}=\sum_{k=1}^{N} D_{i k}^{(1)} D_{k j}^{(m-1)} \text { for } i, j=1,2, \ldots, N
$$

The most convenient method is to pick up the sample points in an equally spaced sample point distribution. Very poor accuracy results are obtained using an equally spaced distribution. Chebyshev-GaussLobatto sample points [4-6], which form an unequally spaced sample point distribution, have been used to improve the calculation accuracy. The unequally spaced sample points of each beam using the Chebyshev-GaussLobatto distribution in the present computation are chosen as:

$$
x_{i}=\frac{1}{2}\left[1-\cos \frac{(i-1) \pi}{N-1}\right] \text { for } i, j=1,2, \ldots, N
$$

\section{ELECTROMECHANICAL BEHAVIOR OF PIEZOELECTRIC LAMINATED COMPOSITE BEAMS}

Consider a piezoelectric composite beam with electric faces normal to thickness $h$. The stresses and electric fields are taken as independent variables in the piezoelectric constitutive equations. The piezoelectric constitutive equation coupled the mechanical and electric fields are given as following equations [33]:

$$
\begin{aligned}
\left\{\begin{array}{l}
\sigma_{11} \\
\sigma_{22} \\
\sigma_{33} \\
\sigma_{23} \\
\sigma_{13} \\
\sigma_{12} \\
D_{1} \\
D_{2} \\
D_{3}
\end{array}\right\}=\left\{\begin{array}{lllllllll}
C_{11} & C_{12} & C_{13} & 0 & 0 & C_{16} & 0 & 0 & e_{31} \\
C_{12} & C_{22} & C_{23} & 0 & 0 & C_{26} & 0 & 0 & e_{32} \\
C_{13} & C_{23} & C_{33} & 0 & 0 & C_{36} & 0 & 0 & e_{33} \\
0 & 0 & 0 & C_{44} & C_{45} & 0 & e_{14} & e_{24} & 0 \\
0 & 0 & 0 & C_{45} & C_{55} & 0 & e_{15} & e_{25} & 0 \\
C_{16} & C_{26} & C_{36} & 0 & 0 & C_{66} & 0 & 0 & 0 \\
0 & 0 & 0 & e_{14} & e_{15} & 0 & \kappa_{11} & \kappa_{12} & 0 \\
0 & 0 & 0 & e_{24} & e_{25} & 0 & \kappa_{12} & \kappa_{22} & 0 \\
e_{31} & e_{32} & e_{33} & 0 & 0 & e_{36} & 0 & 0 & \kappa_{33}
\end{array}\right\} \\
\left\{\begin{array}{l}
\varepsilon_{11} \\
\varepsilon_{22} \\
\varepsilon_{33} \\
\gamma_{23} \\
\gamma_{13} \\
\gamma_{12} \\
E_{1} \\
E_{2} \\
E_{3}
\end{array}\right\}
\end{aligned}
$$

where $C_{i j}$ is the elastic constant, $\varepsilon_{i j}$ is the strain, $\gamma_{i j}$ is engineering shear strain, $\sigma_{i j}$ is the stress, $E_{i}$ is the electric field, $D_{i}$ is the electric displacement and $\kappa_{i j}$ is the dielectric constant measured at a constant stress. A piezoelectric Euler Bernoulli beam is considered and the effect of transverse shear on the deformation of the beam is neglected. The beam is loaded mechanically by application of distributed loads normal to the beam axis, parallel to the beam axis and electrically by application of voltages across the piezoelectric beam. Electric potential can be described as the following equation:

$$
\phi(x, t)=\phi^{(0)}(x, t)+x \phi^{(1)}(x, t)+x^{2} \phi^{(2)}(x, t)
$$

where $\phi$ is the electric potential, $\phi^{(0)}, \phi^{(1)}$ and $\phi^{(2)}$ are the electric potential parameters. A piezoelectric beam subjected a prescribed surface traction $P_{i}$, a surface charge per unit area $\bar{\sigma}$ and a prescribed electrical potential $\bar{\phi}$ on the surface, Hamilton's principle can be described as the following equation [33]:

$$
\begin{aligned}
& \int_{t_{0}}^{t_{1}} \delta\left(\int \frac{1}{2}\left(\varepsilon_{i j} \sigma_{i j}-E_{i} D_{i}-\rho \frac{\partial u}{\partial t}\right) d V\right. \\
& \left.-\int\left(P_{i} u_{i}-\bar{\sigma} \phi\right) d s-\int n_{i} D_{i}(\phi-\bar{\phi}) d s\right) d V=0
\end{aligned}
$$

where $\rho$ is the density of the piezoelectric material, $n_{i}$ is outwardly direct unit normal, $V$ represents the volume of the piezoelectric beam and $s$ stands for the surface. The differential equations governing the piezoelectric laminated composite beams can be expressed as [33]:

$$
\begin{aligned}
& C_{11} A \frac{\partial^{2} u}{\partial x^{2}}+e_{31} A \frac{\partial \phi^{(1)}}{\partial x}-\rho A \frac{\partial^{2} u}{\partial t^{2}}+b P_{x}=0 \\
& C_{11} I \frac{\partial^{4} w}{\partial x^{4}}-2 e_{31} I \frac{\partial^{2} \phi^{(2)}}{\partial x^{2}}+\rho A \frac{\partial^{2} w}{\partial t^{2}}-b P_{z}=0 \\
& \kappa_{11} I \frac{\partial^{2} \phi^{(2)}}{\partial x^{2}}+\kappa_{11} A \frac{\partial^{2} \phi^{(0)}}{\partial x^{2}}+b \bar{\sigma}=0 \\
& e_{31} A \frac{\partial u}{\partial x}+\kappa_{11} I \frac{\partial^{2} \phi^{(1)}}{\partial x^{2}}-\kappa_{33} A \phi^{(1)}+b \bar{\sigma}=0 \\
& 2 e_{31} I \frac{\partial^{2} w}{\partial x^{2}}-\kappa_{11} I \frac{\partial^{2} \phi^{(0)}}{\partial x^{2}}-\kappa_{11} J \frac{\partial^{2} \phi^{(2)}}{\partial x^{2}} \\
& +4 \kappa_{33} I \phi^{(2)}-b \bar{\sigma}=0
\end{aligned}
$$

where $u$ is the deflection parallel to the beam axis, $w$ is the deflection normal to the beam axis, $e_{31}$ is the piezoelectric constant, $\kappa_{11}$ and $\kappa_{33}$ are the dielectric constants, $A$ is the section area, $b$ is the width of the beam, $P_{x}$ is the loads parallel to the beam axis, $P_{z}$ is the loads normal to the beam axis. By employing DQM, equation (1) is substituted into equations (15) to (19). For electromechanical analysis of a fixed-fixed supported piezoelectric beam, the governing differential equation can be discretized in matrix form with respect to the sample 
points as the following equation:

$$
[M]\left\{\frac{\partial^{2} y}{\partial t^{2}}\right\}+[K]\{y\}=\{F\}
$$

where

$$
\left\{\begin{array}{c} 
\\
y\left(x_{1}, t\right) \\
y\left(x_{2}, t\right) \\
: \\
y\left(x_{N}, t\right) \\
y\left(x_{N+1}, t\right) \\
y\left(x_{N+2}, t\right) \\
: \\
y\left(x_{2 N}, t\right) \\
y\left(x_{2 N+1}, t\right) \\
y\left(x_{2 N+2}, t\right) \\
: \\
y\left(x_{3 N}, t\right) \\
y\left(x_{3 N+1}, t\right) \\
y\left(x_{3 N+2}, t\right) \\
: \\
y\left(x_{4 N}, t\right) \\
y\left(x_{4 N+1}, t\right) \\
y\left(x_{4 N+2}, t\right) \\
: \\
y\left(x_{5 N}, t\right)
\end{array}\right\}=\left\{\begin{array}{c}
u\left(x_{1}, t\right) \\
y\left(x_{2}, t\right) \\
: \\
u\left(x_{N}, t\right) \\
w\left(x_{1}, t\right) \\
w\left(x_{2}, t\right) \\
: \\
w\left(x_{N}, t\right) \\
\phi^{(0)}\left(x_{1}, t\right) \\
\phi^{(0)}\left(x_{2}, t\right) \\
: \\
\phi^{(0)}\left(x_{N}, t\right) \\
\phi^{(1)}\left(x_{1}, t\right) \\
\phi^{(1)}\left(x_{2}, t\right) \\
: \\
\phi^{(1)}\left(x_{N}, t\right) \\
\phi^{(2)}\left(x_{1}, t\right) \\
\phi^{(2)}\left(x_{2}, t\right) \\
: \\
\phi^{(2)}\left(x_{N}, t\right)
\end{array}\right\}
$$

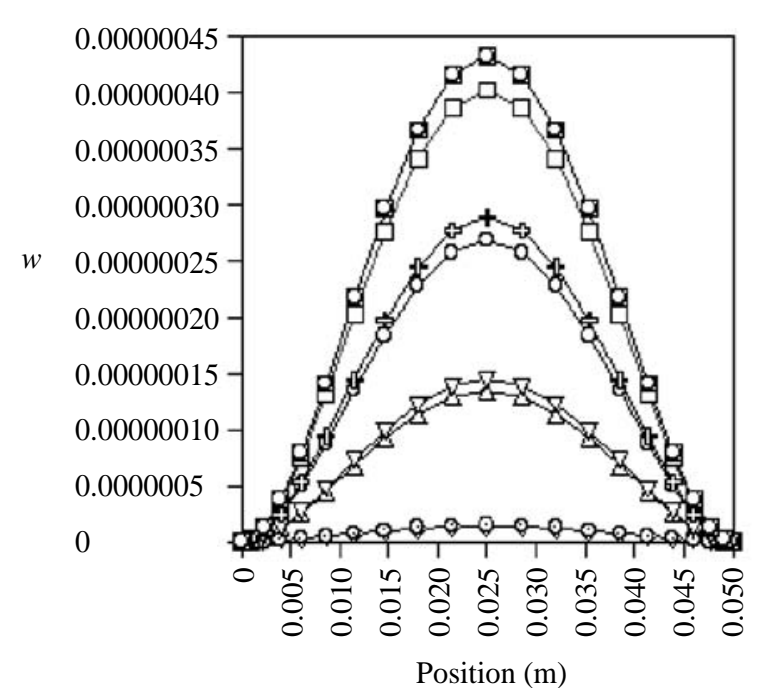

In this study, the electromechanical behavior of the piezoelectric beams is solved using the Wilsonmethod [20]. The Wilson- $\theta$ method assumes that the acceleration terms vary linearly between consecutive sampling instants.

\section{NUMERICAL RESULTS AND DISCUSSION}

The material and the geometric parameters of the piezoelectric laminated composite beams are [33]: $C_{11}=107.6 \times 10^{9} \frac{\mathrm{N}}{\mathrm{m}^{2}}, e_{31}=-9.522 \frac{\mathrm{C}}{\mathrm{m}^{2}}, \kappa_{11}=9.832 \times$ $10^{-9} \frac{\mathrm{F}}{\mathrm{m}}, \kappa_{33}=8.185 \times 10^{-9} \frac{\mathrm{F}}{\mathrm{m}}, b=0.005 \mathrm{~m}, h=0.005 \mathrm{~m}$, and $L=0.05 \mathrm{~m}$. Figures 1,2 , and 3 show the variation of displacement $w$ of piezoelectric beam with various $P_{z}$ and boundary conditions. Three types of boundary condition examples are presented to demonstrate the piezoelectric effect. The first example is a fixed-fixed supported beam subjected a surface traction $P_{z}$. The second example is a fixed-simple supported beam subjected a surface traction $P_{z}$. The third example is a fixed-free supported beam subjected a surface traction $P_{z}$. The boundary conditions influence the elastic displacement of the piezoelectric beam significantly. The fixed-fixed beam deformation shapes are symmetric about the geometric center. The results show that the elastic displacements of the piezoelectric beam are generally smaller than that of the same material without piezoelectric coupling effects. Figures 4, 5 and 6 illustrate the variation of electric potential parameters $\phi^{(0)}$ of fixed-fixed supported beam with various surface tractions $P_{z}$ and boundary conditions. It is interesting to

Fig. 1. The displacement $w$ of fixed-fixed supported piezoelectric beam with various surface tractions $P_{z}$. 


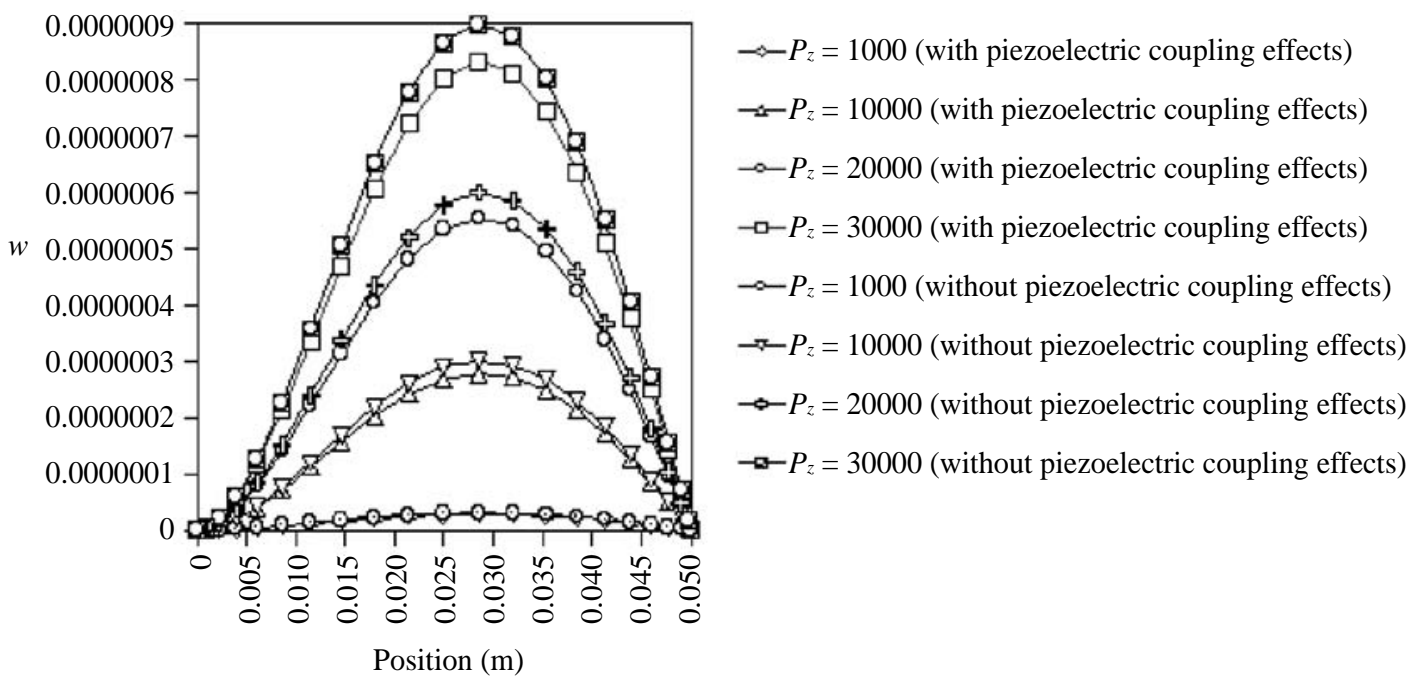

Fig. 2. The displacement $w$ of fixed-simple supported piezoelectric beam with various surface tractions $\boldsymbol{P}_{z}$.

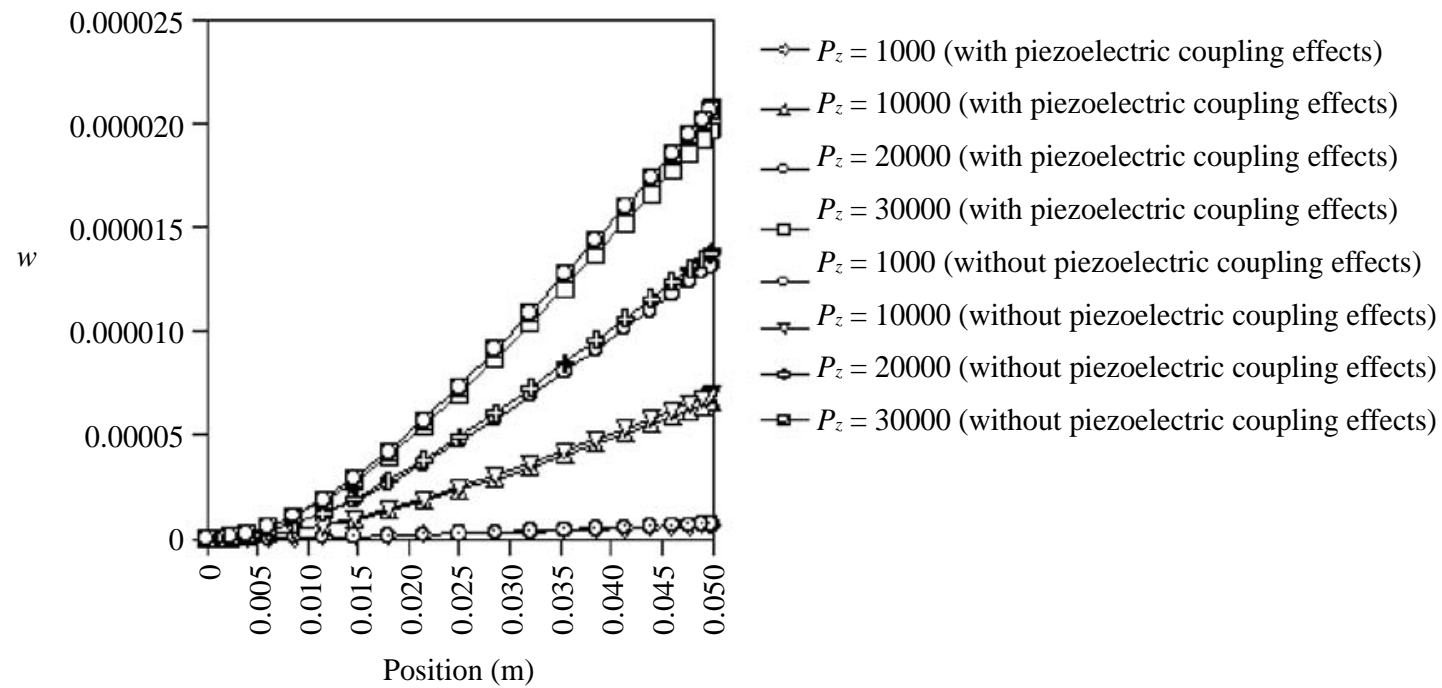

Fig. 3. The displacement $w$ of fixed-free supported piezoelectric beam with various surface tractions $\boldsymbol{P}_{z}$.

study the influence of the different boundary conditions on the voltage response. The boundary conditions influence the electric potential parameters $\phi^{(0)}$ significantly. The electric potential parameters $\phi^{(0)}$ are zero at the boundary points of the piezoelectric beam. This is because the boundary points are ground connection. Figures 7, 8, and 9 reveal the variation of electric potential parameters $\phi^{(2)}$ of piezoelectric beam with various surface tractions $P_{z}$ and boundary conditions. Maximal electric potential parameters $\phi^{(2)}$ are near the center point of the beam for the fixed-fixed supported boundary condition and fixed-simple supported boundary condition. Figure 10 indicates the deflection $w$ at the center point of the piezoelectric beam with surface traction $P_{z}=3000 \sin (2 \pi t / 100)$ and various boundary conditions. In order to characterize the boundary condition effect, three types of boundary condition examples are presented to demonstrate the piezoelectric effect. The results describe the effect of load and boundary conditions on the vibration characteristics of the piezoelectric beam. Figure 11 presents the electric potential parameters $\phi^{(0)}$ at the center point of the piezoelectric beam with surface traction $P_{z}=$ $3000 \sin (2 \pi t / 100)$ and various boundary conditions. Such an effect becomes significant for different boundary conditions. Figure 12 introduces the electric potential parameters $\phi^{(2)}$ at the center point of the piezoelectric beam with surface traction $P_{z}=3000 \sin (2 \pi t / 100)$ 


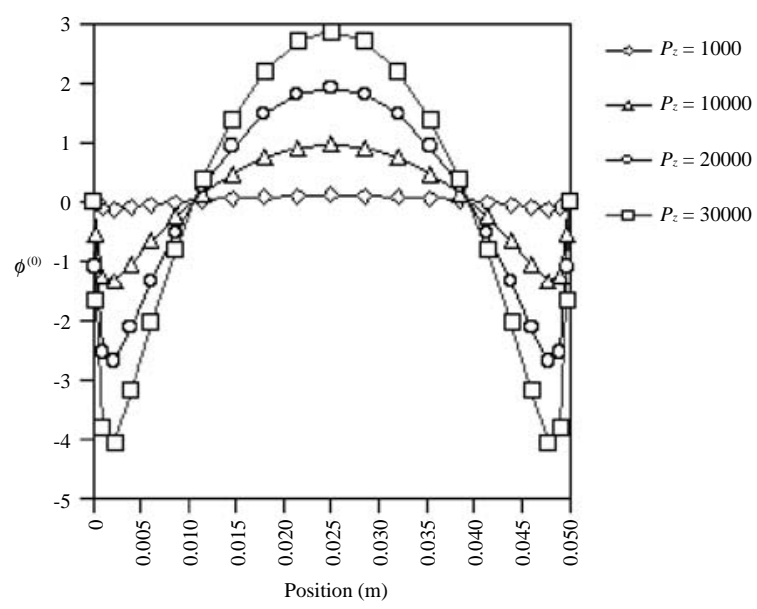

Fig. 4. The electric potential parameters $\phi^{(0)}$ of fixed-fixed supported piezoelectric beam with various surface tractions $\boldsymbol{P}_{z}$.

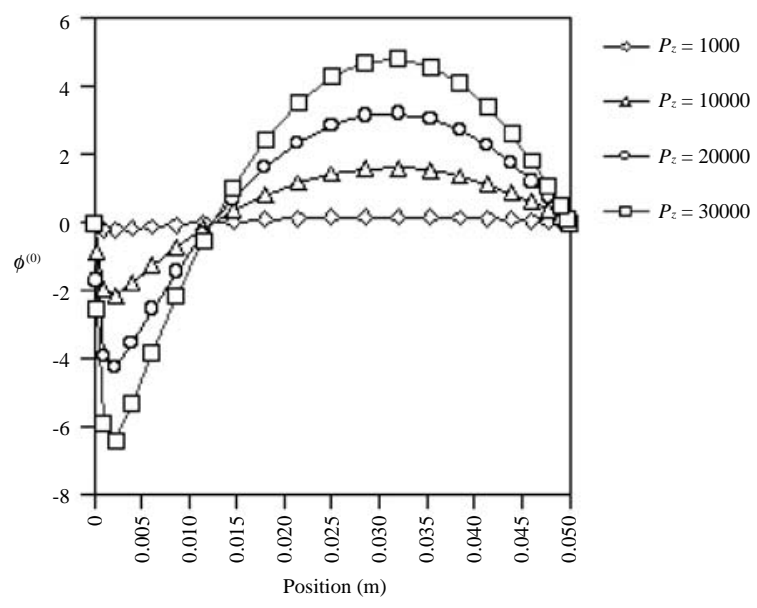

Fig. 5. The electric potential parameters $\phi^{(0)}$ of fixed-simple supported piezoelectric beam with various surface tractions $\boldsymbol{P}_{z}$.

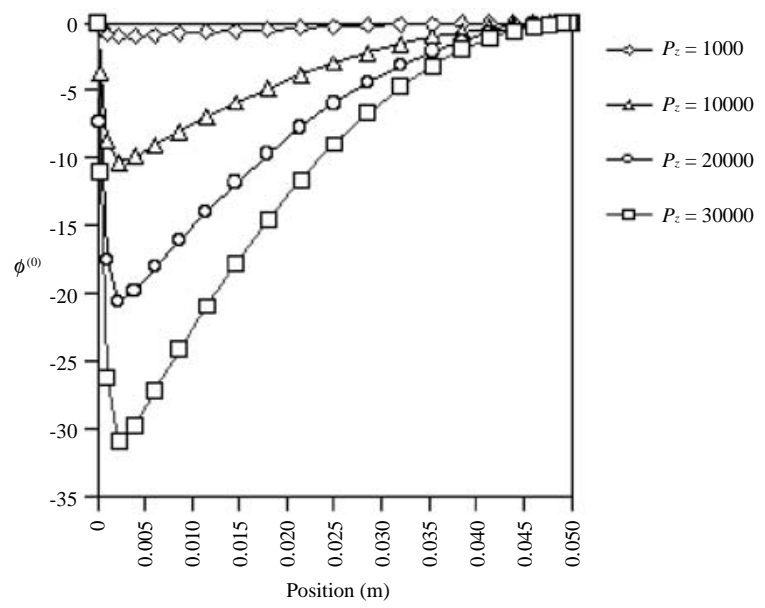

Fig. 6. The electric potential parameters $\phi^{(0)}$ of fixed-free supported piezoelectric beam with various surface tractions $\boldsymbol{P}_{z}$.

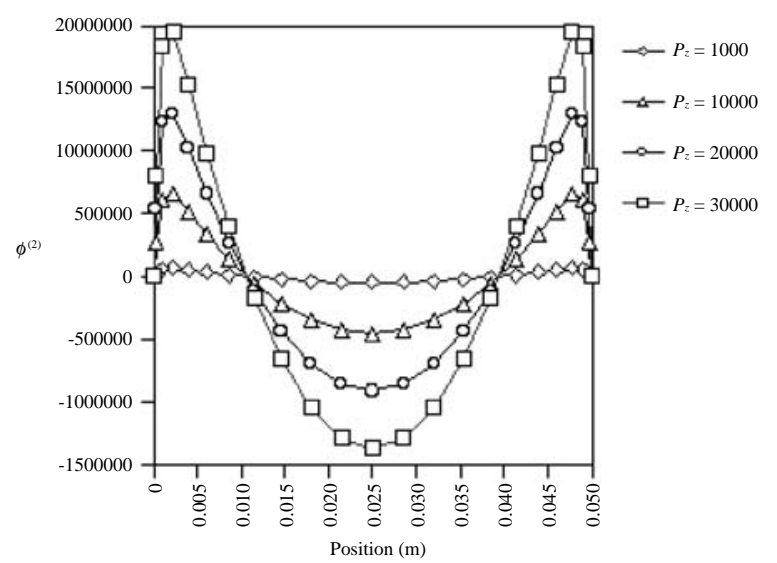

Fig. 7. The electric potential parameters $\phi^{(2)}$ of fixed-fixed piezoelectric supported beam with various surface tractions $P_{z}$.

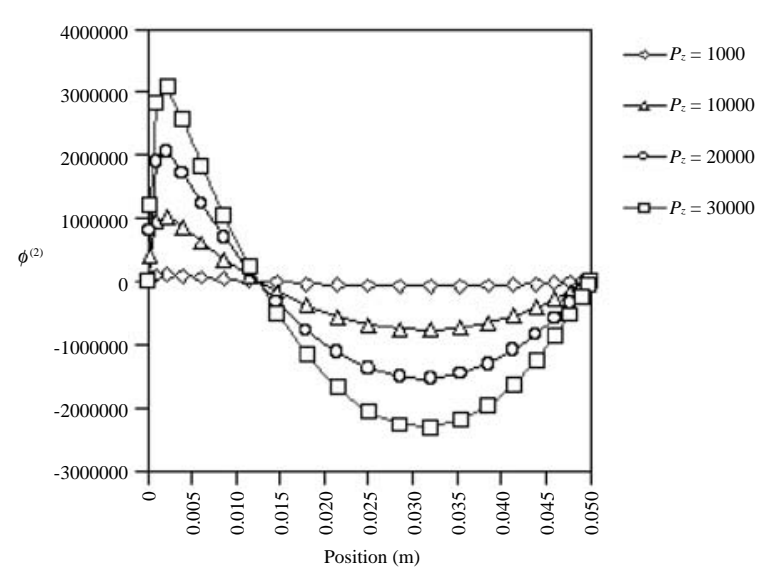

Fig. 8. The electric potential parameters $\phi^{(2)}$ of fixed-simple supported piezoelectric beam with various surface tractions $\boldsymbol{P}_{z}$.

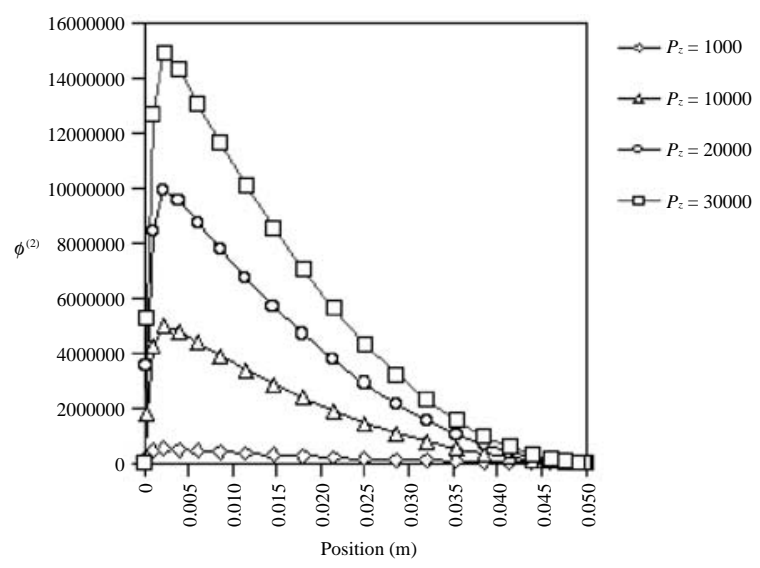

Fig. 9. The electric potential parameters $\phi^{(2)}$ of fixed-free supported piezoelectric beam with various surface tractions $\boldsymbol{P}_{z}$. 


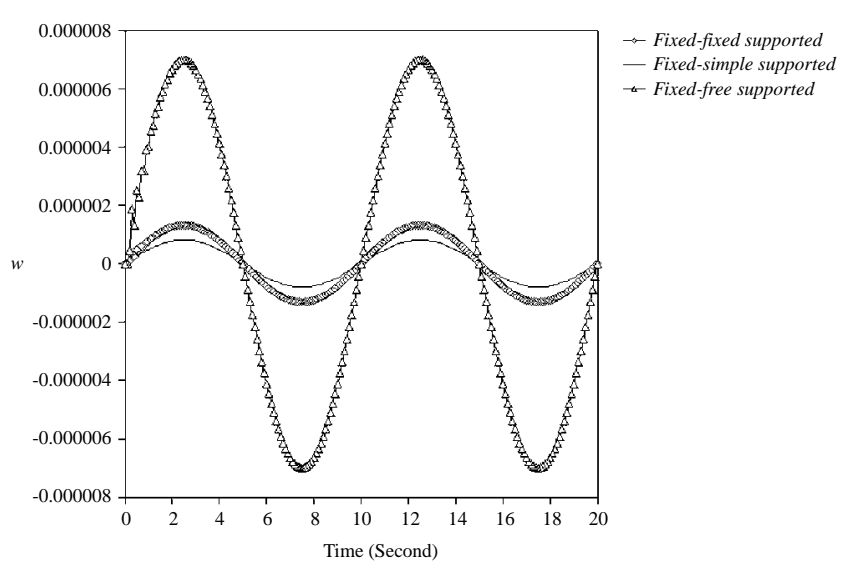

Fig. 10. The deflection $w$ at the center point of the piezoelectric beam with surface traction $P_{z}=3000 \sin (2 \pi t / 100)$ and various boundary conditions.

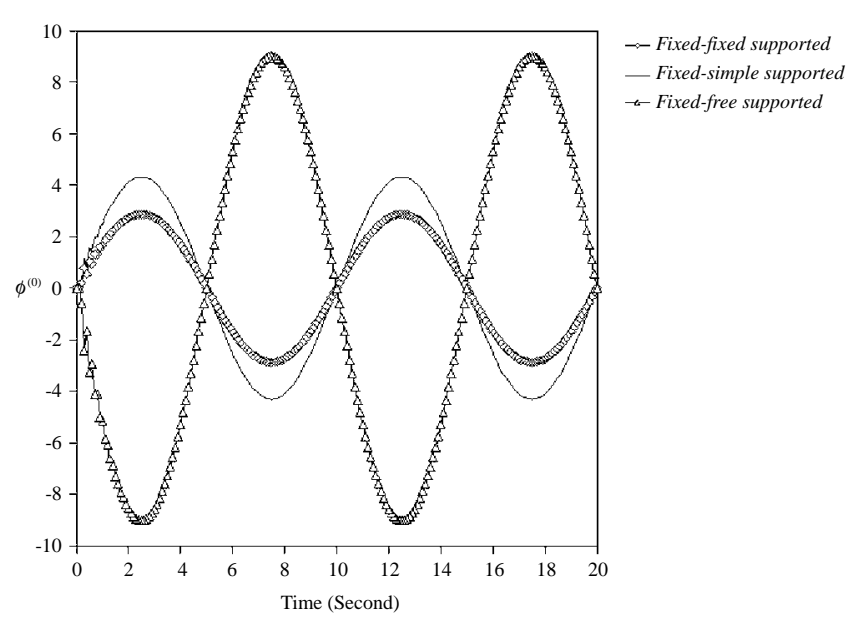

Fig. 11. The electric potential parameters $\phi^{(0)}$ at the center point of the piezoelectric beam with surface traction $P_{z}=3000 \sin (2 \pi t / 100)$ and various boundary conditions.

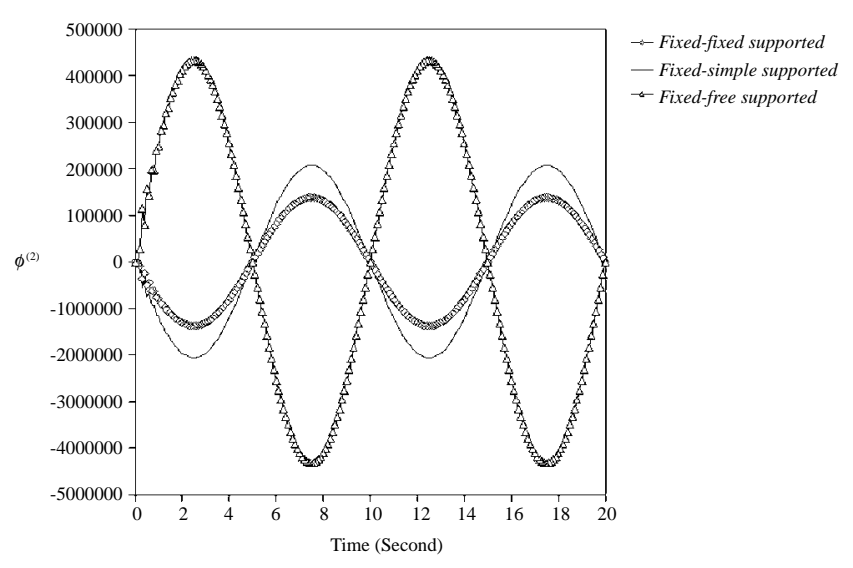

Fig. 12. The electric potential parameters $\phi^{(2)}$ at the center point of the piezoelectric beam with surface traction $P_{z}=3000 \sin (2 \pi t / 100)$ and various boundary conditions. and various boundary conditions. Three types of boundary condition examples are presented to demonstrate the piezoelectric effect.

\section{CONCLUSION}

In this work, the DQM formulation of the electromechanical problems of piezoelectric laminated composite beams as sensors and actuators are proposed and investigated. Using the DQM, the differential equations are transformed into a system of linear algebraic equations. The electromechanical behavior of piezoelectric composite beam with various boundary conditions has been shown. The simplicity of this formulation makes it a desirable candidate for modeling piezoelectric laminated composite beams. The formulation present that the DQM is convenient for solving problems governed by forth or higher order differential equations.

\section{REFERENCES}

1. Bellman, R.E. and Casti, J., "Differential Quadrature and Long-term Integration," J. Math. Anal. Appl., Vol. 34, pp. 235-238 (1971).

2. Bellman, R.E., Kashef, B.G., and Casti, J., "Differential Quadrature: A Technique for Rapid Solution of Nonlinear Partial Differential Equations," J. Comput. Phys., Vol. 10, pp. 40-52 (1972).

3. Bert, C.W., Jang, S.K., and Striz, A.G., "Two New Approximate Methods for Analyzing Free Vibration of Structural Components," AIAA J., Vol. 26, No. 5, pp. 612-618 (1988).

4. Bert, C.W. and Malik, M., "Free Vibration Analysis of Tapered Rectangular Plates by Differential Quadrature Method: a Semi-analytical Approach," J. Sound Vib., Vol. 190, No. 1, pp. 41-63 (1996).

5. Bert, C.W. and Mailk, M., "Differential Quadrature Method in Computational Mechanics: A Review," Appl. Mech. Rev., Vol. 49, No. 1, pp. 1-28 (1996).

6. Bert, C.W. and Malik, M., "On the Relative Effects of Transverse Shear Deformation and Rotary Inertia on the Free Vibration of Symmetric Cross-ply Laminated Plates," J. Sound Vib., Vol. 193, No. 4, pp. 927-933 (1996).

7. Bert, C.W., Wang, X., and Striz, A.G., "Differential Quadrature for Static and Free Vibration Analysis of Anisotropic Plates," Int. J. Solids. Struct., Vol. 30, pp. 1737-1744 (1993).

8. Bert, C.W., Wang, X., and Striz, A.G., "Convergence of the DQ Method in the Analysis of Anisotropic Plates," $J$. Sound Vib., Vol. 170, pp. 140-144 (1994).

9. Chen, C.N., "The Warping Torsion Bar Model of the Differential Quadrature Element Method," Comput. 
Struct., Vol. 66, No. 2-3, pp. 249-257 (1998).

10. Chen, L.W., Lin, C.Y., and Wang, C.C., "Dynamic Stability Analysis and Control of a Composite Beam with Piezoelectric Layers," Compos. Struct., Vol. 56, pp. 97-109 (2002).

11. Chen, W.L., Striz, A.G., and Bert, C.W., "High-accuracy Plane Stress and Plate Elements in the Quadrature Element Method," Int. J. Solid Struct., Vol. 37, pp. 627647 (2000).

12. Choi, S.T., Wu, J.D., and Chou, J.D., "Dynamic Analysis of a Spinning Timoshenko Beam by the Differential Quadrature Method," AIAA J., Vol. 38, No. 5, pp.851856 (2000).

13. Du, H., Liew, K.M., and Lim, M.K., "Generalized Differential Quadrature Method for Buckling Analysis," $J$. Eng. Mech., Vol. 122, No. 2, pp. 95-100 (1996).

14. Feng, Y. and Bert, C.W., "Application of the Quadrature Method to Flexural Vibration Analysis of a Geometrically Nonlinear Beam," Nonlinear Dynam., Vol. 3, pp. 13-18 (1992).

15. Huang, J.F. and Dai, W.L., "Static and Dynamic Electromechanical Response of Piezoelectric Transducers," Mater. Lett., Vol. 50, pp. 209-218 (2001).

16. Jang, S.K., Bert, C.W., and Striz, A.G., "Application of Differential Quadrature to Static Analysis of Structural Components," Int. J. Numer. Eng., Vol. 28, pp. 561-577 (1989).

17. Liew, K.M., Han, J.B., and Xiao, Z.M., "Differential Quadrature Method for Thick Symmetric Cross-ply Laminates with First-order Shear Flexibility," Int. J. Solids Struct., Vol. 33, No. 18, pp. 2647-2658 (1996).

18. Liew, K.M., Han, J.B., Xiao, Z.M., and Du, H., "Differential Quadrature Method for Mindlin Plates on Winkler Foundations," Int. J. Mech. Sci., Vol. 38, No. 4, pp. 405421 (1996).

19. Malik, M. and Bert, C.W., "Implementing Multiple Boundary Conditions in the DQ Solution of Higherorder PDE's Application to Free Vibration of Plates," Int. J. Numer. Eng., Vol. 39, pp. 1237-1258 (1996).

20. Ozkul, T.A., "A Finite Element Formulation for Dynamic Analysis of Shells of General Shape by Using the Wilson- $\theta$ method," Thin-Wall. Struct., Vol. 42, pp. 497-513 (2004).

21. Quan, J.R. and Chang, C.T., "New Insights in Solving Distributed System Equations by the Quadrature Method-I. Analysis," Comput. Chem. Eng., Vol. 13, pp. 779-788 (1989).
22. Quan, J.R. and Chang, C.T., "New Insights in Solving Distributed System Equations by the Quadrature MethodII. Numerical Experiments," Comput. Chem. Eng., Vol. 13, pp. 1017-1024 (1989).

23. Saitoh, K. and Koshiba, M., "Stress Analysis Method Considering Piezoelectric Effects and its Application to Static Strain Optic Devices," J. Lightwave Technol., Vol. 17, No. 9, pp. 1626-1633 (1999).

24. Sherbourne, A.N. and Pandey, M.D., "Differential Quadrature Method in the Buckling Analysis of Beams and Composite Plates," Comput. Struct., Vol. 40, pp. 903-913 (1991).

25. Shu, C. and Du, H., "Free Vibration Analysis of Laminated Composite Cylindrical Shells by DQM," Composites Part B, Vol. 28B, pp. 267-274 (1997).

26. Striz, A.G., Chen, W., and Bert, C.W., "Static Analysis of Structures by the Quadrature Element Method (QEM)," Int. J. Solids Struct., Vol. 31, pp. 2807-2818 (1994).

27. Sun, J. and Zhu, Z., "Upwind Local Differential Quadrature Method for Solving Incompressible Viscous Flow," Comput. Method. Appl. M., Vol. 188, pp. 495504 (2000).

28. Tadmor, E.B. and Kosa, G., "Electromechanical Coupling Correction for Piezoelectric Layered Beams," J. Microelectromech. S., Vol. 12, No. 6, pp. 899-906 (2003).

29. Tanaka, M. and Chen, W., "Dual Reciprocity BEM Applied to Transient Elastodynamic Problems with Differential Quadrature Method in Time," Comput. Method Appl. M., Vo. 190, pp. 2331-2347 (2001).

30. Tomasiello, S., "Differential Quadrature Method: Application to Initial-Boundary-Value Problems," $J$. Sound Vib., Vol. 218, No. 4, pp. 573-585 (1998).

31. Wang, X. and Bert, C.W., "A New Approach in Applying Differential Quadrature to Static and Free Vibrational Analyses of Beams and Plates," J. Sound Vib., Vol. 162, No. 3, pp. 566-572 (1993).

32. Weinberg, M.S., "Working Equations for Piezoelectric Actuators and Sensors," J. Microelectromech. S., Vol. 8, pp. 529-533 (1999).

33. Wu, J.F., "Analysis for Piezoelectric Sensors and Actuators," Master Thesis, Department of Mechanical Engineering, Feng Chia University, Taiwan (2000).

34. Yang, R.B. and Huang, J.H., "Fracture Analysis of Piezoelectric Materials with Flat Ellipsoidal Crack," Materials Letters, Vol. 57, pp. 481-489 (2002). 